\title{
Nonparametric Spherical Topic Modeling with Word Embeddings
}

\author{
Nematollah Kayhan Batmanghelich* Ardavan Saeedi* \\ CSAIL, MIT \\ kayhan@mit.edu
}

\author{
Karthik R. Narasimhan \\ CSAIL, MIT \\ karthiknemit.edu
}

\author{
Samuel J. Gershman \\ Department of Psychology \\ Harvard University \\ gershmanefas.harvard.edu
}

\begin{abstract}
Traditional topic models do not account for semantic regularities in language. Recent distributional representations of words exhibit semantic consistency over directional metrics such as cosine similarity. However, neither categorical nor Gaussian observational distributions used in existing topic models are appropriate to leverage such correlations. In this paper, we propose to use the von Mises-Fisher distribution to model the density of words over a unit sphere. Such a representation is well-suited for directional data. We use a Hierarchical Dirichlet Process for our base topic model and propose an efficient inference algorithm based on Stochastic Variational Inference. This model enables us to naturally exploit the semantic structures of word embeddings while flexibly discovering the number of topics. Experiments demonstrate that our method outperforms competitive approaches in terms of topic coherence on two different text corpora while offering efficient inference. ${ }^{1}$
\end{abstract}

\section{Introduction}

Prior work on topic modeling has mostly involved the use of categorical likelihoods (Blei et al., 2003; Blei and Lafferty, 2006; Rosen-Zvi et al., 2004). Applications of topic models in the textual domain treat words as discrete observations, ignoring the semantics of the language. Recent developments in distributional representations of words (Mikolov et al., 2013; Pennington et al.,

\footnotetext{
${ }^{*}$ Authors contributed equally and listed alphabetically.

${ }^{1}$ Code is available at https://github.com/ Ardavans/sHDP.
}

2014) have succeeded in capturing certain semantic regularities, but have not been explored extensively in the context of topic modeling. In this paper, we propose a probabilistic topic model with a novel observational distribution that integrates well with directional similarity metrics.

One way to employ semantic similarity is to use the Euclidean distance between word vectors, which reduces to a Gaussian observational distribution for topic modeling (Das et al., 2015). The cosine distance between word embeddings is another popular choice and has been shown to be a good measure of semantic relatedness (Mikolov et al., 2013; Pennington et al., 2014). The von Mises-Fisher (vMF) distribution is well-suited to model such directional data (Dhillon and Sra, 2003; Banerjee et al., 2005) but has not been previously applied to topic models.

In this work, we use vMF as the observational distribution. Each word can be viewed as a point on a unit sphere with topics being canonical directions. More specifically, we use a Hierarchical Dirichlet Process (HDP) (Teh et al., 2006), a Bayesian nonparametric variant of Latent Dirichlet Allocation (LDA), to automatically infer the number of topics. We implement an efficient inference scheme based on Stochastic Variational Inference (SVI) (Hoffman et al., 2013).

We perform experiments on two different English text corpora: 20 NEWSGROUPS and NIPS and compare against two baselines - HDP and Gaussian LDA. Our model, spherical HDP (sHDP), outperforms all three systems on the measure of topic coherence. For instance, sHDP obtains gains over Gaussian LDA of $97.5 \%$ on the NiPS dataset and $65.5 \%$ on the 20 NEWSGROUPS dataset. Qualitative inspection reveals consistent topics produced by sHDP. We also empirically demonstrate that employing SVI leads to efficient 
topic inference.

\section{Related Work}

Topic modeling and word embeddings Das et al. (2015) proposed a topic model which uses a Gaussian distribution over word embeddings. By performing inference over the vector representations of the words, their model is encouraged to group words that are semantically similar, leading to more coherent topics. In contrast, we propose to utilize von Mises-Fisher (vMF) distributions which rely on the cosine similarity between the word vectors instead of euclidean distance.

vMF in topic models The vMF distribution has been used to model directional data by placing points on a unit sphere (Dhillon and Sra, 2003). Reisinger et al. (2010) propose an admixture model that uses vMF to model documents represented as vector of normalized word frequencies. This does not account for word level semantic similarities. Unlike their method, we use vMF over word embeddings. In addition, our model is nonparametric.

Nonparametric topic models HDP and its variants have been successfully applied to topic modeling (Paisley et al., 2015; Blei, 2012; He et al., 2013); however, all these models assume a categorical likelihood in which the words are encoded as one-hot representation.

\section{Model}

In this section, we describe the generative process for documents. Rather than one-hot representation of words, we employ normalized word embeddings (Mikolov et al., 2013) to capture semantic meanings of associated words. Word $n$ from document $d$ is represented by a normalized $M$ dimensional vector $x_{d n}$ and the similarity between words is quantified by the cosine of angle between the corresponding word vectors.

Our model is based on the Hierarchical Dirichlet Process (HDP). The model assumes a collection of "topics" that are shared across documents in the corpus. The topics are represented by the topic centers $\mu_{k} \in \mathbb{R}^{M}$. Since word vectors are normalized, the $\mu_{k}$ can be viewed as a direction on unit sphere. Von Mises-Fisher (vMF) is a distribution that is commonly used to model directional data. The likelihood of the topic $k$ for word $x_{d n}$

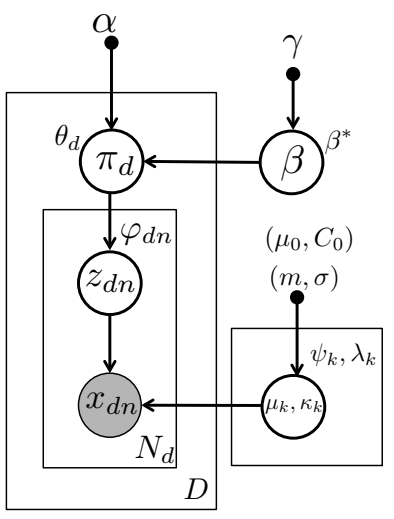

Figure 1: Graphical representation of our spherical HDP (sHDP) model. The symbol next to each random variable denotes the parameter of its variational distribution. We assume $D$ documents in the corpus, each document contains $N_{d}$ words and there are countably infinite topics represented by $\left(\mu_{k}, \kappa_{k}\right)$.

is:

$$
f\left(x_{d n} ; \mu_{k} ; \kappa_{k}\right)=\exp \left(\kappa_{k} \mu_{k}^{T} x_{d n}\right) C_{M}\left(\kappa_{k}\right)
$$

where $\kappa_{k}$ is the concentration of the topic $k$, the $C_{M}\left(\kappa_{k}\right):=\kappa_{k}^{M / 2-1} /\left((2 \pi)^{M / 2} I_{M / 2-1}\left(\kappa_{k}\right)\right)$ is the normalization constant, and $I_{\nu}(\cdot)$ is the modified Bessel function of the first kind at order $\nu$. Interestingly, the log-likelihood of the vMF is proportional to $\mu_{k}^{T} x_{d n}$ (up to a constant), which is equal to the cosine distance between two vectors. This distance metric is also used in Mikolov et al. (2013) to measure semantic proximity.

When sampling a new document, a subset of topics determine the distribution over words. We let $z_{d n}$ denote the topic selected for the word $n$ of document $d$. Hence, $z_{d n}$ is drawn from a categorical distribution: $z_{d n} \sim \operatorname{Mult}\left(\pi_{d}\right)$, where $\pi_{d}$ is the proportion of topics for document $d$. We draw $\pi_{d}$ from a Dirichlet Process which enables us to estimate the the number of topics from the data. The generative process for the generation of new document is as follows:

$$
\begin{array}{ll}
\beta \sim \operatorname{GEM}(\gamma) & \pi_{d} \sim \operatorname{DP}(\alpha, \beta) \\
\kappa_{k} \sim \log -\operatorname{Normal}\left(m, \sigma^{2}\right) & \mu_{k} \sim \operatorname{vMF}\left(\mu_{0}, C_{0}\right) \\
z_{d n} \sim \operatorname{Mult}\left(\pi_{d}\right) & x_{d n} \sim \operatorname{vMF}\left(\mu_{k}, \kappa_{k}\right)
\end{array}
$$

where $\operatorname{GEM}(\gamma)$ is the stick-breaking distribution with concentration parameter $\gamma, \operatorname{DP}(\alpha, \beta)$ is a Dirichlet process with concentration parameter $\alpha$ and stick proportions $\beta$ (Teh et al., 2012). We use 
log-normal and vMF as hyper-prior distributions for the concentrations $\left(\kappa_{k}\right)$ and centers of the topics $\left(\mu_{k}\right)$ respectively. Figure 1 provides a graphical illustration of the model.

Stochastic variational inference In the rest of the paper, we use bold symbols to denote the variables of the same kind (e.g., $\quad \boldsymbol{x}_{d}=\left\{x_{d n}\right\}_{n}$, $\left.\boldsymbol{z}:=\left\{z_{d n}\right\}_{d, n}\right)$. We employ stochastic variational mean-field inference (SVI) (Hoffman et al., 2013) to estimate the posterior distributions of the latent variables. SVI enables us to sequentially process batches of documents which makes it appropriate in large-scale settings.

To approximate the posterior distribution of the latent variables, the mean-field approach finds the optimal parameters of the fully factorizable $q$ (i.e., $q(\mathbf{z}, \beta, \boldsymbol{\pi}, \boldsymbol{\mu}, \boldsymbol{\kappa}):=q(\mathbf{z}) q(\beta) q(\boldsymbol{\pi}) q(\boldsymbol{\mu}) q(\boldsymbol{\kappa}))$ by maximizing the Evidence Lower Bound (ELBO),

$$
\mathcal{L}(q)=\mathbb{E}_{q}[\log p(\boldsymbol{X}, \mathbf{z}, \beta, \boldsymbol{\pi}, \boldsymbol{\mu}, \boldsymbol{\kappa})]-\mathbb{E}_{q}[\log q]
$$

where $\mathbb{E}_{q}[\cdot]$ is expectation with respect to $q$, $p(\boldsymbol{X}, \mathbf{z}, \beta, \boldsymbol{\pi}, \boldsymbol{\mu}, \boldsymbol{\kappa})$ is the joint likelihood of the model specified by the HDP model.

The variational distributions for $\boldsymbol{z}, \boldsymbol{\pi}, \boldsymbol{\mu}$ have the following parametric forms,

$$
\begin{aligned}
q(\mathbf{z}) & =\operatorname{Mult}(\mathbf{z} \mid \boldsymbol{\varphi}) \\
q(\boldsymbol{\pi}) & =\operatorname{Dir}(\boldsymbol{\pi} \mid \boldsymbol{\theta}) \\
q(\boldsymbol{\mu}) & =\operatorname{vMF}(\boldsymbol{\mu} \mid \boldsymbol{\psi}, \boldsymbol{\lambda}),
\end{aligned}
$$

where Dir denotes the Dirichlet distribution and $\boldsymbol{\varphi}, \boldsymbol{\theta}, \boldsymbol{\psi}$ and $\boldsymbol{\lambda}$ are the parameters we need to optimize the ELBO. Similar to (Bryant and Sudderth, 2012), we view $\beta$ as a parameter; hence, $q(\beta)=\delta_{\beta^{*}}(\beta)$. The prior distribution $\kappa$ does not follow a conjugate distribution; hence, its posterior does not have a closed-form. Since $\kappa$ is only one dimensional variable, we use importance sampling to approximate its posterior. For a batch size of one (i.e., processing one document at time), the update equations for the parameters are:

$$
\begin{aligned}
& \begin{aligned}
\varphi_{d w k} \propto \exp \left\{\mathbb{E}_{q}\left[\log \operatorname{vMF}\left(x_{d w} \mid \psi_{k}, \lambda_{k}\right)\right]\right. \\
\left.\quad+\mathbb{E}_{q}\left[\log \pi_{d k}\right]\right\}
\end{aligned} \\
& \theta_{d k} \leftarrow(1-\rho) \theta_{d k}+\rho\left(\alpha \beta_{k}+D \sum_{n=1}^{W} \omega_{w j} \varphi_{d w k}\right) \\
& t \leftarrow(1-\rho) t+\rho s\left(\boldsymbol{x}_{d}, \varphi_{d k}\right) \\
& \psi \leftarrow t /\|t\|_{2}, \quad \lambda \leftarrow\|t\|_{2}
\end{aligned}
$$

where $D, \omega_{w j}, W, \rho$ are the total number of documents, number of word $w$ in document $j$, the total number of words in the dictionary, and the step size, respectively. $t$ is a natural parameter for $\mathrm{vMF}$ and $s\left(\boldsymbol{x}_{d}, \varphi_{d k}\right)$ is a function computing the sufficient statistics of vMF distribution of the topic $k$. We use numerical gradient ascent to optimize for $\beta^{*}$. For exact forms of $\mathbb{E}_{q} \log \left[\operatorname{vMF}\left(x_{d w} \mid \psi_{k}, \lambda_{k}\right)\right]$ and $\mathbb{E}_{q}\left[\log \pi_{d k}\right]$, see Appendix.

\section{Experiments}

Setup We perform experiments on two different text corpora: 11266 documents from 20 NEWSGROUPS $^{2}$ and 1566 documents from the NIPS corpus $^{3}$. We utilize 50-dimensional word embeddings trained on text from Wikipedia using word2vec ${ }^{4}$. The vectors are normalized to have unit $\ell^{2}$-norm, which has been shown to provide superior performance (Levy et al., 2015)).

We evaluate our model using the measure of topic coherence (Newman et al., 2010), which has been shown to effectively correlate with human judgement (Lau et al., 2014). For this, we compute the Pointwise Mutual Information (PMI) using a reference corpus of 300k documents from Wikipedia. The PMI is calculated using cooccurence statistics over pairs of words $\left(u_{i}, u_{j}\right)$ in 20-word sliding windows:

$$
\operatorname{PMI}\left(u_{i}, u_{j}\right)=\log \frac{p\left(u_{i}, u_{j}\right)}{p\left(u_{i}\right) \cdot p\left(u_{j}\right)}
$$

Additionally, we also use the metric of normalized PMI (NPMI) to evaluate the models in a similar fashion:

$$
\operatorname{NPMI}\left(u_{i}, u_{j}\right)=\frac{\log \frac{p\left(u_{i}, u_{j}\right)}{p\left(u_{i}\right) \cdot p\left(u_{j}\right)}}{-\log p\left(u_{i}, u_{j}\right)}
$$

We compare our model with two baselines: HDP and the Gaussian LDA model. We ran G-LDA with various number of topics $(k)$.

Results Table 2 details the topic coherence averaged over all topics produced by each model. We observe that our sHDP model outperforms GLDA by 0.08 points on 20 NEWSGROUPS and by 0.17 points in terms of PMI on the NIPS dataset. The NPMI scores also show a similar trend with sHDP obtaining the best scores on both datasets. We can also see that the individual topics inferred

\footnotetext{
${ }^{2}$ http: //qwone.com/ jason/20Newsgroups/

${ }^{3}$ http: //www. cs.nyu. edu/ roweis/data. html

${ }^{4}$ https://code.google.com/p/word2vec/
} 


\begin{tabular}{|c|c|c|c|c|c|c|c|}
\hline \multicolumn{8}{|c|}{ Gaussian LDA } \\
\hline vector & shows & network & hidden & performance & net & figure & size \\
\hline image & feature & learning & term & work & references & shown & average \\
\hline gaussian & show & model & rule & press & introduction & neurons & present \\
\hline equation & motion & neural & word & tion & statistical & point & family \\
\hline generalization & action & input & means & ing & related & large & versus \\
\hline images & spike & data & words & eq & comparison & neuron & spread \\
\hline gradient & series & function & approximate & performed & source & small & median \\
\hline theory & final & time & derived & em & statistics & fig & physiology \\
\hline dimensional & robot & set & describe & vol & free & cells & children \\
\hline 1.16 & 0.4 & 0.35 & 0.29 & 0.25 & 0.25 & 0.21 & 0.2 \\
\hline \multicolumn{8}{|c|}{ Spherical HDP } \\
\hline neural & function & analysis & press & pattern & problem & noise & algorithm \\
\hline layer & linear & theory & cambridge & fig & process & gradient & error \\
\hline neurons & functions & computational & journal & temporal & method & propagation & parameters \\
\hline neuron & vector & statistical & vol & shape & optimal & signals & computation \\
\hline activation & random & field & eds & smooth & solution & frequency & algorithms \\
\hline brain & probability & simulations & trans & surface & complexity & feedback & compute \\
\hline cells & parameter & simulation & springer & horizontal & estimation & electrical & binary \\
\hline cell & dimensional & nonlinear & volume & vertical & prediction & filter & mapping \\
\hline synaptic & equation & dynamics & review & posterior & solve & detection & optimization \\
\hline 1.87 & 1.73 & 1.51 & 1.44 & 1.41 & 1.19 & 1.12 & 1.03 \\
\hline
\end{tabular}

Table 1: Examples of top words for the most coherent topics (column-wise) inferred on the NIPS dataset by Gaussian LDA $(\mathrm{k}=40)$ and Spherical HDP. The last row for each model is the topic coherence (PMI) computed using Wikipedia documents as reference.

\begin{tabular}{|c|cc|cc|}
\hline & \multicolumn{4}{|c|}{ Topic Coherence } \\
Model & \multicolumn{2}{|c|}{ 20 NEws } & \multicolumn{2}{c|}{ NIPS } \\
& pmi & npmi & pmi & npmi \\
\hline HDP & 0.037 & 0.014 & 0.270 & 0.062 \\
G-LDA (k=10) & -0.061 & -0.006 & 0.214 & 0.055 \\
G-LDA (k=20) & -0.017 & 0.001 & 0.215 & 0.052 \\
G-LDA (k=40) & 0.052 & 0.015 & 0.248 & 0.057 \\
G-LDA (k=60) & 0.082 & 0.021 & 0.137 & 0.034 \\
sHDP & $\mathbf{0 . 1 6 2}$ & $\mathbf{0 . 0 4 6}$ & $\mathbf{0 . 4 4 2}$ & $\mathbf{0 . 1 0 2}$ \\
\hline
\end{tabular}

Table 2: Average topic coherence for various baselines (HDP, Gaussian LDA (G-LDA)) and sHDP. $k=$ number of topics. Best scores are shown in bold.

by sHDP make sense qualitatively and have higher coherence scores than G-LDA (Table 1). This supports our hypothesis that using the vMF likelihood helps in producing more coherent topics. SHDP produces 16 topics for the 20 NEWSGROUPS and 92 topics on the NIPS dataset.

Figure 2 shows a plot of normalized loglikelihood against the runtime of sHDP and GLDA. ${ }^{5}$ We calculate the normalized value of loglikelihood by subtracting the minimum value from it and dividing it by the difference of maximum

\footnotetext{
${ }^{5}$ Our sHDP implementation is in Python and the G-LDA code is in Java.
}

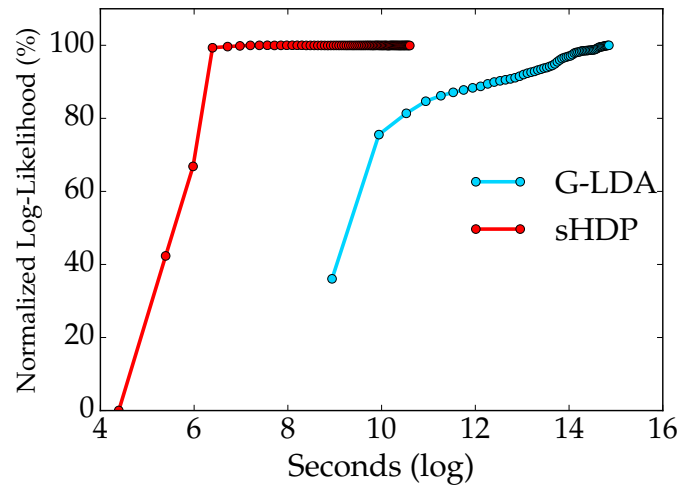

Figure 2: Normalized log-likelihood (in percentage) over a training set of size 1566 documents from the NIPS corpus. Since the log-likelihood values are not comparable for the Gaussian LDA and the sHDP, we normalize them to demonstrate the convergence speed of the two inference schemes for these models.

and minimum values. We can see that SHDP converges faster than G-LDA, requiring only around five iterations while G-LDA takes longer to converge.

\section{Conclusion}

Classical topic models do not account for semantic regularities in language. Recently, distributional 
representations of words have emerged that exhibit semantic consistency over directional metrics like cosine similarity. Neither categorical nor Gaussian observational distributions used in existing topic models are appropriate to leverage such correlations. In this work, we demonstrate the use of the von Mises-Fisher distribution to model words as points over a unit sphere. We use HDP as the base topic model and propose an efficient algorithm based on Stochastic Variational Inference. Our model naturally exploits the semantic structures of word embeddings while flexibly inferring the number of topics. We show that our method outperforms three competitive approaches in terms of topic coherence on two different datasets.

\section{Acknowledgments}

Thanks to Rajarshi Das for helping with the Gaussian LDA experiments and Matthew Johnson for his help with the HDP code.

\section{References}

Arindam Banerjee, Inderjit S Dhillon, Joydeep Ghosh, and Suvrit Sra. 2005. Clustering on the unit hypersphere using von mises-fisher distributions. In Journal of Machine Learning Research, pages 13451382 .

David M Blei and John D Lafferty. 2006. Dynamic topic models. In Proceedings of the 23rd international conference on Machine learning, pages 113120. ACM.

David M Blei, Andrew Y Ng, and Michael I Jordan. 2003. Latent dirichlet allocation. the Journal of machine Learning research, 3:993-1022.

David M Blei. 2012. Probabilistic topic models. Communications of the ACM, 55(4):77-84.

Michael Bryant and Erik B Sudderth. 2012. Truly nonparametric online variational inference for hierarchical dirichlet processes. In Advances in Neural Information Processing Systems, pages 2699-2707.

Rajarshi Das, Manzil Zaheer, and Chris Dyer. 2015. Gaussian LDA for topic models with word embeddings. In Proceedings of the 53nd Annual Meeting of the Association for Computational Linguistics.

Inderjit S Dhillon and Suvrit Sra. 2003. Modeling data using directional distributions. Technical report, Technical Report TR-03-06, Department of Computer Sciences, The University of Texas at Austin. URL ftp://ftp. cs. utexas. edu/pub/techreports/tr0306. ps. gz.

Siddarth Gopal and Yiming Yang. 2014. Von misesfisher clustering models.
Yulan He, Chenghua Lin, Wei Gao, and Kam-Fai Wong. 2013. Dynamic joint sentiment-topic model. ACM Transactions on Intelligent Systems and Technology (TIST), 5(1):6.

Matthew D Hoffman, David M Blei, Chong Wang, and John Paisley. 2013. Stochastic variational inference. The Journal of Machine Learning Research, 14(1):1303-1347.

Matthew Johnson and Alan Willsky. 2014. Stochastic variational inference for bayesian time series models. In Proceedings of the 31st International Conference on Machine Learning (ICML-14), pages 18541862.

Jey Han Lau, David Newman, and Timothy Baldwin. 2014. Machine reading tea leaves: Automatically evaluating topic coherence and topic model quality. In $E A C L$, pages 530-539.

Omer Levy, Yoav Goldberg, and Ido Dagan. 2015. Improving distributional similarity with lessons learned from word embeddings. Transactions of the Association for Computational Linguistics, 3:211-225.

Tomas Mikolov, Ilya Sutskever, Kai Chen, Greg S Corrado, and Jeff Dean. 2013. Distributed representations of words and phrases and their compositionality. In Advances in neural information processing systems, pages 3111-3119.

David Newman, Jey Han Lau, Karl Grieser, and Timothy Baldwin. 2010. Automatic evaluation of topic coherence. In Human Language Technologies: The 2010 Annual Conference of the North American Chapter of the Association for Computational Linguistics, pages 100-108. Association for Computational Linguistics.

John Paisley, Chingyue Wang, David M Blei, and Michael I Jordan. 2015. Nested hierarchical dirichlet processes. Pattern Analysis and Machine Intelligence, IEEE Transactions on, 37(2):256-270.

Jeffrey Pennington, Richard Socher, and Christopher D. Manning. 2014. Glove: Global vectors for word representation. In Empirical Methods in Natural Language Processing (EMNLP), pages 15321543.

Joseph Reisinger, Austin Waters, Bryan Silverthorn, and Raymond J Mooney. 2010. Spherical topic models. In Proceedings of the 27th International Conference on Machine Learning (ICML-10), pages 903-910.

Michal Rosen-Zvi, Thomas Griffiths, Mark Steyvers, and Padhraic Smyth. 2004. The author-topic model for authors and documents. In Proceedings of the 20th conference on Uncertainty in artificial intelligence, pages 487-494. AUAI Press.

Yee Whye Teh, Michael I Jordan, Matthew J Beal, and David M Blei. 2006. Hierarchical dirichlet processes. Journal of the American Statistical Association, 101:1566-1581. 
Yee Whye Teh, Michael I Jordan, Matthew J Beal, and David M Blei. 2012. Hierarchical dirichlet processes. Journal of the american statistical association.

\section{Appendinx}

\section{Mean field update equations}

In this section, we provide the mean field update equations. The SVI update equations can be derived from the mean field update (Hoffman et al., 2013).

The following term is computed for the update equations:

$$
\begin{aligned}
& \mathbb{E}_{q}\left[\log \operatorname{vMF}\left(x_{d n} \mid \mu_{k}, \kappa_{k}\right)\right]=\mathbb{E}_{q}\left[\log C_{M}\left(\kappa_{k}\right)\right]+ \\
& \mathbb{E}_{q}\left[\kappa_{k}\right] x_{d n}^{T} \mathbb{E}_{q}\left[\mu_{k}\right]
\end{aligned}
$$

where $C_{M}(\cdot)$ is explained in Section 3. The difficulty here lies in computing $\mathbb{E}_{q}\left[\kappa_{k}\right]$ and $\mathbb{E}_{q}\left[C_{M}\left(\kappa_{k}\right)\right]$. However, $\kappa$ is a scalar value. Hence, to compute $\mathbb{E}_{q}\left[\kappa_{k}\right]$, we divide a reasonable interval of $\kappa_{k}$ into grids and compute the weight for each grid point as suggested by Gopal and Yang (2014):

$$
\begin{aligned}
p\left(\kappa_{k} \mid \cdots\right) & \propto \exp \left(n_{k} \log C_{M}\left(\kappa_{k}\right)+\right. \\
& \left.\kappa_{k}\left(\sum_{d=1}^{D} \sum_{n=1}^{N_{d}}\left[\varphi_{d n}\right]_{k}\left\langle x_{d n}, \mathbb{E}_{q}\left[\mu_{k}\right]\right\rangle\right)\right) \times \\
& \log \operatorname{Normal}\left(\kappa_{k} \mid m, \sigma^{2}\right)
\end{aligned}
$$

where $n_{k}=\sum_{d=1}^{D} \sum_{d=1}^{N_{d}}\left[\varphi_{d n}\right]_{k}$ and $[a]_{k}$ denotes the $k$ 'th element of vector $a$. After computing the normalized weights, we can compute $\mathbb{E}_{q}\left[\kappa_{k}\right]$ or expectation of any other function of $\kappa_{k}$ (e.g., $\left.\mathbb{E}_{q}\left[C_{M}\left(\kappa_{k}\right)\right]\right)$. The rest of the terms can be computed as follows:

$$
\begin{aligned}
\mathbb{E}_{q}\left[\mu_{k}\right] & =\mathbb{E}_{q}\left[\frac{I_{M / 2}\left(\kappa_{k}\right)}{I_{M / 2-1}\left(\kappa_{k}\right)}\right] \psi_{k}, \\
\psi_{k} & =\mathbb{E}_{q}\left[\kappa_{k}\right]\left(\sum_{d=1}^{D} \sum_{n=1}^{N_{d}}\left[\varphi_{d n}\right]_{k} x_{d n}\right)+C_{0} \mu_{0} \\
\psi_{k} & \leftarrow \frac{\psi_{k}}{\left\|\psi_{k}\right\|_{2}}, \\
{\left[\mathbb{E}_{q}\left[\log \left(\pi_{d}\right)\right]\right]_{k} } & =\Psi\left(\left[\theta_{d}\right]_{k}\right)-\Psi\left(\sum_{k}\left[\theta_{d}\right]_{k}\right), \\
{\left[\varphi_{d n}\right]_{k} } & \propto \exp \left(\mathbb{E}_{q}\left[\log \operatorname{vMF}\left(x_{d n} \mid \mu_{k}, \kappa_{k}\right)\right]+\mathbb{E}_{q}\left[\log \left(\left[\pi_{d}\right]_{k}\right)\right]\right), \\
{\left[\theta_{d}\right]_{k}=} & \alpha+\sum_{n=1}^{N_{d}}\left[\varphi_{d n}\right]_{k}
\end{aligned}
$$

$\Psi(\cdot)$ is the digamma function.
To find $\beta^{*}$, similar to Johnson and Willsky (2014), we use the gradient expression of ELBO with respect to $\beta$ and take a truncated gradient step on $\beta$ ensuring $\beta^{*} \geq 0$. 